\title{
INVARIANT SUBSPACE OF STRICTLY SINGULAR OPERATORS
}

\author{
RUAN JI-SHOU
}

(Communicated by John B. Conway)

\begin{abstract}
In this paper, we show that strictly singular operators are condensing maps. Moreover, we obtain a new result that every bounded linear operator $T$ on a Banach space that commutes with a nonzero strictly singular operator $S$ has a non-trivial invariant closed subspace.
\end{abstract}

\section{INTRODUCTION}

The famous problem that has challenged mathematicians for many years is whether every bounded linear operator on a Banach space has a non-trivial closed invariant subspace. Except for a few special classes of operators (such as the self-adjoint operators on a Hilbert space), it was unsolved for general Banach spaces until 1954, when N. Aronszajn and K. Smith proved that every compact operator has a non-trivial closed subspace. Twelve years later, A. Bernstein and A. Robinson, using the method of nonstandard analysis, extended this result to an operator $T$ such that $p(T)$ is compact for some nonzero polynomial $p(t)$. Still later on, in 1973, V. I. Lomonosov published a remarkable generalization of the Bernstein-Robinson Theorem (see [2]). In this paper, we use the method of non-linear functional analysis to extend this result to an operator $T$ commuting with a strictly singular operator $S$.

We denote the set of all linear bounded operators from a normed space $X$ to a normed space $Y$ by $B(X, Y)$, the set of all strictly singular operators by $S(X, X)$ (see [4]), the set of all elements of $B(X, X)$ commuting with an operator $B$ by $\mathfrak{U}_{B}$, the convex hull of a set $A$ by $\operatorname{co}(A)$, and the closure of $A$ by $\bar{A} . x \in A-B$ means $x \in A$ and $x \notin B$.

Let us begin with some concepts and properties that we shall meet in this paper. Most of them are well known and a few unfamiliar ones are referred to [3, §§16.1 and 16.2].

Received by the editors June 19, 1989.

1980 Mathematics Subject Classification (1985 Revision). Primary 47A15; Secondary 47H09.

Key words and phrases. Invariant subspace, strictly singular operator, fixed point theorem, condensing map, measure of noncompactness, partition of unity, paracompact, Zorn's Lemma, Tychonoff's Theorem. 
Each metric space $X$ is paracompact and for each open covering of $X$ there is a partition of unity subordinated to it.

Let $A$ be a bounded subset of a metric space $X$. We put $\gamma(A)=\inf \left\{d:\right.$ There is a finite number of subsets $S_{i}$ such that $\bigcup_{i=1}^{n} S_{i} \supset A$, and $\left.\operatorname{diam}\left(S_{i}\right) \leq d, i=1, \ldots, n.\right\}$.

$\gamma(A)$ is called the measure of noncompactness of $A$, and it has the following properties:

1. $\gamma(A)=0$ iff $\bar{A}$ is a compact set;

2. for each scalar $t, \gamma(t A)=|t| \gamma(A) ; \gamma(\bar{A})=\gamma(A), \gamma(\operatorname{co}(A))=\gamma(A)$;

3. for arbitrary bounded subsets $A, B$ of $X$, $\gamma(A+B) \leq \gamma(A)+\gamma(B), \gamma(A \cup B)=\max (\gamma(A), \gamma(B))$, and $\gamma(A) \leq \gamma(B)$ if $A \subset B$.

Further, let $X, Y$ be metric spaces, and $F: X \rightarrow Y$ be a bounded continuous map. We call $F$ a condensing map if $\gamma(F(A))<\gamma(A)$ for each bounded subset of $X$ with $\gamma(A) \neq 0$. We call a bounded linear operator $T: X \rightarrow Y$ strictly singular if the restriction of $T$ to any infinite-dimensional subspace of $X$ is not an isomorphism (see [4]).

According to [3, Theorem 16.12], we have the following fact:

Let $X$ be a Banach space and $A$ an open subset of $X$. Let $F: \bar{A} \rightarrow X$ be a continuous bounded map satisfying the following conditions:

(i) There is $x_{0} \in A$ such that $F(x)-x_{0} \neq \alpha\left(x-x_{0}\right)$ for each $x \in \partial A$ and $\alpha>1$;

(ii) $F$ is a condensing map.

Then $F$ has at least one fixed point $\bar{x} \in \bar{A}$.

\section{MAIN RESUlt}

Lemma 1. Let $B$ be the balanced hull of $A$; then $\gamma(B)=\gamma(A)$.

Proof. Since $B=\bigcup_{|z| \leq 1}(z A)$, for each $\varepsilon>0$, we can find $z_{1}, \ldots, z_{n}$ such that, for $|z| \leq 1$, there is $z_{i}$ satisfying $\left|z-z_{i}\right|<\varepsilon$. Put

$$
D_{i}=\left\{z:|z| \leq 1,\left|z-z_{i}\right|<\varepsilon\right\} .
$$

Then

$$
\gamma\left(\bigcup_{z \in D_{i}}(z A)\right) \leq \gamma\left(z_{i} A\right)+\varepsilon \gamma(A) \leq \gamma(A)+\varepsilon \gamma(A),
$$

and $B=\bigcup_{i=1}^{n}\left(\bigcup_{z \in D_{i}}(z A)\right)$. So $\gamma(B)=\max \left\{\gamma\left(\bigcup_{z \in D_{i}}(z A)\right), i=1, \ldots, n\right\} \leq$ $\gamma(A)+\varepsilon \gamma(A)$, that is, $\gamma(B) \leq \gamma(A)$. Clearly, $\gamma(A) \leq \gamma(B)$; thus we have $\gamma(B)=\gamma(A)$.

Lemma 2. Let $X, Y$ be Banach spaces and $T \in B(X, Y)$. The following statements are equivalent:

(i) $T$ is a one to one map and there is a constant $C>0$ such that $\gamma(T(D)) \geq$ $C \gamma(D)$ for each bounded subset $D$ of $X$.

(ii) $T: X \rightarrow Y$ is an isomorphism. 
Proof. (i) $\Rightarrow$ (ii). Assume that $T$ is not an isomorphism. There is a sequence in $S_{x}$, the closed unit sphere in $X$, such that $\lim \left\|T x_{n}\right\|=0$. We see that ${\overline{\left\{x_{n}\right\}_{n=1}^{\infty}}}_{n}^{\infty}$ is not a compact set. In fact, if there is $x_{0} \in S_{x}$ such that $x_{n_{k}} \rightarrow x_{0}$, we would have $\left\|T x_{0}\right\|=\lim \left\|T x_{n_{k}}\right\|=0$, which is contrary to the assumption. Therefore $\gamma\left(\left\{x_{n}\right\}_{n=1}^{\infty}\right) \neq 0$. However, $\overline{\left\{T x_{n}\right\}_{n=1}^{\infty}}$ is a compact set and so $\gamma\left(\left\{T x_{n}\right\}_{n=1}^{\infty}\right)=0$. This again is contrary to the assumption.

(ii) $\Rightarrow$ (i). Let $D$ be a bounded subset of $X$ with $\gamma(D) \neq 0$. For any finite number of subsets $S_{i}$ of $Y$ with $\bigcup_{i} S_{i} \supset T D$ and $\operatorname{diam}\left(S_{i}\right) \leq d$ for all $i$, we have $\bigcup_{i} T^{-1}\left(S_{i}\right) \supset D$, and $\operatorname{diam}\left(T^{-1}\left(S_{i}\right)\right) \leq\left\|T^{-1}\right\| d$, and hence $d \geq\left\|T^{-1}\right\|^{-1} \gamma(D)$. By definition we have $\gamma(T(D)) \geq\left\|T^{-1}\right\|^{-1} \gamma(D)$. For $\gamma(D)=0$, (i) follows trivially from (ii).

Lemma 3. Let $X$ be a normed space and $T \in B(X, X)$. Let $D$ be a subset of $X$ satisfying the conditions:

(i) For each pair $x, y \in D, x \neq y$ implies $T x \neq T y$;

(ii) $\gamma(T(D)) \geq \gamma(D)$.

Then $\gamma\left(D_{1}\right) \leq \gamma\left(T\left(D_{1}\right)\right)$ for each subset $D_{1}$ of $D$.

Proof. The conclusion is trivial if $\gamma(D)=0$. We prove it for $\gamma(D) \neq 0$. For each $x \in D-D_{1}$ with $D_{1} \subset D$ properly, we have $D=(D-\{x\}) \cup\{x\}$ and $T D=T(D-\{x\}) \cup\{T x\}$.

Further, $\gamma(D-\{x\}) \leq \gamma(T(D-\{x\}))$ and $\gamma(D-\{x\}) \neq 0$, since $\gamma(D-\{x\})=$ $\gamma(D)$. We put

$$
W=\left\{V: D_{1} \subset V \subset D, \gamma(T(V)) \geq \gamma(V)\right\}
$$

Clearly, $W$ is nonempty. We define a partial order relation on $W$ as follows: For $V_{1}, V_{2} \in W, V_{1}<V_{2}$ iff $V_{1} \supset V_{2}$ properly.

For any totally ordered nonempty subset $M$ of $W$, put $V_{0}=\bigcap_{v \in M} V$. We assert that $V_{0}$ is an upper bound of $M$. Actually, we have $\inf _{v \in M} \gamma(T V) \geq$ $\gamma\left(T V_{0}\right)$, $\inf _{v \in M} \gamma(V) \geq \gamma\left(V_{0}\right)$, and $\inf _{v \in M} \gamma(T V) \geq \inf _{v \in M} \gamma(V)$. We need only to show $\gamma\left(T V_{0}\right)=\inf _{v \in M} \gamma(T V)$. In fact, if otherwise, we let $\varepsilon_{0}=$ $\inf _{v \in M} \gamma(T V)-\gamma\left(T V_{0}\right)>0$. Then we have $\gamma\left(T\left(V-V_{0}\right)\right) \geq \gamma\left(T V_{0}\right)+\varepsilon_{0} \geq \varepsilon_{0}$ for all $V \in M$. Since $M \neq \varnothing$ and is totally ordered, so is the set $\left\{T\left(V-V_{0}\right): V \in\right.$ $M\}$. It follows that $\gamma\left(\bigcap_{v \in M}\left(T\left(V-V_{0}\right)\right)\right) \neq 0$ since $\gamma\left(T\left(V-V_{0}\right)\right) \geq \varepsilon_{0}$ for all $V \in M$. By the assumption (i), $\bigcap_{v \in M}\left(T\left(V-V_{0}\right)\right)=T\left(\bigcap_{v \in M}\left(V-V_{0}\right)\right)=\varnothing$, which is impossible; i.e. $\gamma\left(T V_{0}\right)=\inf _{v \in M} \gamma(T V)$. It follows that $\gamma\left(T V_{0}\right) \geq$ $\gamma\left(V_{0}\right)$. So $V_{0}$ is an upper bound of $M$.

By Zorn's Lemma, there exists a maximal element $D_{0}$ in $W$. We see that $D_{0}=D_{1}$. Otherwise, there would be an $x \in D_{0}-D_{1}$, and $D_{0}-\{x\} \in W$. This is contrary to the maximality of $D_{0}$ in $W$.

Lemma 4. Let $X, Y$ be Banach spaces and $T \in B(X, Y)$. If $\gamma(A) \leq \gamma(T A)$ holds for each bounded subset $A$ of $X$, then $\operatorname{diam} T^{-1}(0)<+\infty$. 
Proof. Assume that $\operatorname{diam} T^{-1}(0)=+\infty$. Then the closed unit sphere in $T^{-1}(0)$ is not totally bounded, and we can find an infinite sequence $\left\{x_{n}\right\}_{n=1}^{\infty}$ in this sphere contained in $T^{-1}(0)$ which has no Cauchy subsequence, and hence ${\overline{\left\{x_{n}\right.}}_{n=1}^{\infty}$ is not compact. Then $\gamma\left(\left\{x_{n}\right\}_{n=1}^{\infty}\right) \neq 0$, but $\gamma\left(\left\{T x_{n}\right\}_{n=1}^{\infty}\right)=0$. This is contrary to the assumption.

Theorem 1. Let $X$ be a Banach space and $T \in B(X, X)$. Then $T \in S(X, X)$ implies that $T$ is a condensing map.

Proof. Suppose $T$ is not condensing; there would be a bounded subset $D$ of $X$ with $\gamma(D) \neq 0$, such that $\gamma(T D) \geq \gamma(D)$. We may choose a subset of $D$, say $D_{1}$, such that $\gamma\left(D_{1}\right) \neq 0, \gamma\left(T D_{1}\right) \geq \gamma\left(D_{1}\right)$ and $T x \neq T y$ for all $x, y \in D_{1}$ when $x \neq y$.

Let $A$ be the absolutely convex hull of $D_{1} \cup\{0\}$; then $A$ satisfies the conditions (i) and (ii) of Lemma 3 . Since $\bar{A}$ is an absolutely convex neighborhood in $Z=\overline{\operatorname{span}(A)}$, then for each bounded subset $G$ of $Z$, there exists a constant $\lambda>0$ such that $\lambda \bar{A} \supset G$. It follows that $\gamma(T G) \geq \gamma(G)$ from Lemma 3 and $\gamma(T(\lambda \bar{A})) \geq \gamma(\lambda \bar{A})$.

We put $T_{1}=\left.T\right|_{Z}$, the restriction of $T$ to $Z$. Since $G$ is arbitrary, we see that $\operatorname{dim} T_{1}^{-1}(0)<+\infty$ by Lemma 4 . There is a closed subspace $Z_{1}$ of $Z$, that is complementary to $T_{1}^{-1}(0)$, i.e. $Z=T_{1}^{-1}(0) \oplus Z_{1}$. Thus $\left.T\right|_{Z_{1}}=\left.T_{1}\right|_{Z_{1}}$ is injective. Therefore, $\left.T\right|_{Z_{1}}$ is an isomorphism by Lemma 2 . This is contrary to the fact that $T$ is a strictly singular operator.

Theorem 2. Let $T$ belong to $B(X, X)$ and commute with a nonzero element $S$ of $S(X, X)$. If $\left\{\mathfrak{U}_{T} x\right\}$ is dense in $X$ for each nonzero element $x$ of $X$, there exists an element $T_{0}$ of $\mathfrak{U}_{T}$ such that 1 is an eigenvalue of $T_{0} S$.

Proof. Since $S \neq 0$, we may choose $x_{0} \in X$ with $\delta=\left\|S x_{0}\right\|-\|S\|>0$. Let $G=\left\{x:\left\|x-x_{0}\right\|<1\right\}$, and $\bar{G}=\left\{x:\left\|x-x_{0}\right\|<1\right\}$. We have then $\|S x\|>0$ for all $x \in \bar{G}$. By the assumption $\left\{\mathfrak{U}_{T} y\right\}$ is dense in $X$ for all $y \in S \bar{G}$. Hence there is a $T_{y} \in \mathfrak{U}_{T}$ such that $\left\|T_{y} y-x_{0}\right\|<1$. Let $\varepsilon_{y}=$ $\left(1-\left\|T_{y} y-x_{0}\right\|\right) /\left\|T_{y}\right\|>0$. Then $\left\|T_{y} z-x_{0}\right\|<1$ for all $z$ in $O\left(y, \varepsilon_{y}\right)$, where $O\left(y, \varepsilon_{y}\right)=\left\{z:\|y-z\|<\varepsilon_{y}\right\}$.

The family $\left\{O\left(y, \varepsilon_{y}\right): y \in S \bar{G}\right\}$ is an open covering of $\overline{S \bar{G}}$. Let $\left\{f_{y}: y \in\right.$ $S \bar{G}\}$ be a partition of unity subordinated to $\left\{O\left(y, \varepsilon_{y}\right): y \in S \bar{G}\right\}$, and let

$$
\varphi(z)=\sum_{y \in S \bar{G}} f_{y}(z) T_{y} z \quad \text { for all } z \in S \bar{G} .
$$

Then $\varphi$ is a bounded continuous map from $S \bar{G}$ to $\bar{G}$. Again, let

$$
\psi(x)=\sum_{y \in S \bar{G}} f_{y}(S x) T_{y} S x \quad \text { for all } x \in \bar{G} .
$$

Then $\psi$ is a bounded continuous map from $\bar{G}$ to $\bar{G}$. 
For each $x \in \partial G$, we have the following estimate:

$$
\left\|\psi(x)-x_{0}\right\|=\left\|\sum_{y \in S \bar{G}} f_{y}(S x) T_{y} S x-x_{0}\right\|=\left\|\sum^{\prime} f_{y}(S x)\left(T_{y} S x-x_{0}\right)\right\|<1,
$$

where $\sum^{\prime}$ means the sum of the terms in which $S x$ belongs to the support of $f_{y}$. We have $\psi(x)-x_{0} \neq \alpha\left(x-x_{0}\right)$ for each $x \in \partial G$ and all $\alpha>1$.

Moreover, for each bounded subset $G_{1}$ of $G$ with $\gamma\left(G_{1}\right) \neq 0$, we show that $\gamma\left(\psi\left(G_{1}\right)\right)<\gamma\left(G_{1}\right)$. In fact, let us put $U_{y}=\overline{f_{y}\left(S G_{1}\right)}$. Then $U_{y}$ is a compact set and the product $\prod_{y \in S \bar{G}} U_{y}$ is compact in $\prod_{y \in S \bar{G}} E_{y}$ by Tychonoff's Theorem, where all $E_{y}$ are one-dimensional Euclidean spaces.

For each $x \in G_{1}, f_{y}(S x)=0$ for all but finitely many $y$, say $y_{1}, \ldots, y_{n}$, such that $f_{y_{i}}(S x) \neq 0, i=1, \ldots, n$. For arbitrary $\varepsilon>0$, we put

$$
U_{i}=\left\{r: r \in E_{1},\left|f_{y_{i}}(S x)-r\right|<\varepsilon / 2^{i}\right\} \text {. }
$$

Then $U(x)=U_{1} \times U_{2} \cdots \times U_{n} \times \prod_{y \in S \bar{G}-\left\{y_{1}, \ldots, y_{n}\right\}} E_{y}$ is an open set and $\{U(x)$ : $\left.x \in G_{1}\right\}$ is an open covering of $\prod_{y \in S \bar{G}} U_{y}$. Therefore, we may choose $x_{1}, \ldots$, $x_{m}$ such that $\left\{U\left(x_{i}\right): i=1, \ldots, m\right\}$ is also an open covering. We put

$$
G_{1 i}=\left\{x: x \in G_{1},\left\{f_{y}(S x)\right\}_{y \in S \bar{G}} \in U\left(x_{i}\right)\right\}, \quad i=1, \ldots, m .
$$

Then $G_{1}=G_{11} \cup G_{12} \cup \cdots \cup G_{1 m}$. We assert that

$$
\bigcup_{x \in G}\left\{\sum_{y \in S \bar{G}} f_{y}(S x)\left(T_{y} S\right) x\right\} \subset \sum_{y \in S \bar{G}} f_{y}\left(S x_{i}\right)\left(T_{y} S\right)\left(G_{1 i}\right)+C \varepsilon O(0,1)
$$

for some constant $C>0$. In fact, for each $x \in G_{1 i}$, we have $\sum_{j=1}^{n} \mid f_{y}(S x)-$ $f_{y_{j}}\left(S x_{i}\right) \mid<\varepsilon$, hence

$$
\sum_{y \in S \bar{G}-\left\{y_{1}, \ldots, y_{n}\right\}} f_{y}(S x)<\varepsilon
$$

and

$$
\sum_{y \in S \bar{G}-\left\{y_{1}, \ldots, y_{n}\right\}} f_{y}(S x) T_{y} S x \in \varepsilon G
$$

This implies that

$$
\sum_{y \in S \bar{G}} f_{y}(S x) T_{y} S x \in \sum_{y \in S \bar{G}} f_{y}\left(S x_{i}\right) T_{y} S x+\varepsilon O(0,1)+\varepsilon G .
$$

Furthermore,

$$
\bigcup_{x \in G_{1 i}}\left\{\sum_{y \in S \bar{G}} f_{y}(S x) T_{y} S x\right\} \subset \sum_{y \in S \bar{G}} f_{y}\left(S x_{i}\right)\left(T_{y} S\right)\left(G_{1 i}\right)+C \varepsilon O(0,1),
$$

where $C=2\left(1+\left\|x_{0}\right\|\right)$. 
From the preceding arguments, we obtain

$$
\begin{aligned}
\gamma\left(\psi\left(G_{1}\right)\right) & \leq \gamma\left(\bigcup_{j=1}^{m}\left(\sum_{y \in S \bar{G}} f_{y}\left(S x_{i}\right) T_{y} S\left(G_{1 j}\right)\right)+C \varepsilon O(0,1)\right) \\
& \leq \max \left\{\gamma\left(\sum_{y \in S \bar{G}} f_{y}\left(S x_{i}\right) T_{y} S\left(G_{1 j}\right)\right)+2 C \varepsilon: j=1, \ldots, m\right\} \\
& \leq \max \left\{\sum_{y \in S \bar{G}} f_{y}\left(S x_{i}\right) \gamma\left(T_{y} S\left(G_{1 j}\right)\right)+2 C \varepsilon: j=1, \ldots, m\right\} \\
& <\max \left\{\sum_{y \in S \bar{G}} f_{y}\left(S x_{i}\right) \gamma\left(G_{1 j}\right)+2 C \varepsilon: j=1, \ldots, m\right\} \\
& =\max \left\{\gamma\left(G_{1 j}\right)+2 C \varepsilon: j=1, \ldots, m\right\} \leq \gamma\left(G_{1}\right)+2 C \varepsilon .
\end{aligned}
$$

By the arbitrariness of $\varepsilon$, we have $\gamma\left(\psi\left(G_{1}\right)\right)<\gamma\left(G_{1}\right)$ when $\gamma\left(G_{1}\right) \neq 0$. We get a fixed point $\bar{x}$ of $\psi$ in $\bar{G}$, that is $\psi(\bar{x})=\bar{x}$. Let $T_{0}=\sum_{y \in S \bar{G}} f_{y}(S \bar{x}) T_{y}$; then $T_{0} \in \mathfrak{U}_{T}$. Clearly, 1 is an eigenvalue of $T_{0} S$.

Theorem 3. Let $T$ belong to $B(X, X)$ and $T \neq \alpha I$. If $T$ commutes with a nonzero strictly singular operator $S$, then there is a nontrivial closed subspace of $X$ that is invariant under each operator in $\mathfrak{U}_{T}$.

The proof of this theorem is completely similar to that of Lomonosov's Theorem and is omitted here.

\section{ACKNOWLEDGMENTS}

I am especially grateful to Professors Chen Wen-Yan and Chen Jian-Gang whose critical suggestions led to the substantial improvement of this paper. I am also indebted to my colleague Na Qi-Yan who gave the proof of Lemma 2. I further wish to thank Professor Wang Tseng-Yi for his revision of the manuscript.

\section{REFERENCES}

1. H. Radjavi and P. Rosenthal, Invariant subspaces, Springer, Berlin-Heidelberg, New York, 1974.

2. A. E. Taylor and D. C. Lay, Introduction to functional analysis, 2nd ed., New York, Chichester, Brisbane, Toronto, 1980.

3. C. Wen-Yan, Nonlinear functional analysis, (Chinese edition), Gansu Renmin Press, 1981, pp. 232-251.

4. J. Lindenstrauss and L. Tzafriri, Classical Banach spaces I, Springer-Verlag, BerlinHeidelberg, New York, 1977, pp. 75-77.

5. A. Wilanski, Modern methods in topological vector spaces, McGraw-Hill International Book Company, New York, 1978.

6. J. Dugundji, Topology, Allyn and Bacon, Inc., Boston, 1966.

Department of Mathematics, Xinjiang University, Urumqi, China 